\title{
THE MATING BEHAVIOR OF EURYCOTIS FLORIDANA (WALKER) (BLATTARIA, BLATTOIDEA, BLATTIDAE, POLYZOSTERIINAE) ${ }^{1}$
}

\author{
By Robert H. Barth, JR. ${ }^{2}$ \\ Department of Zoology \\ The University of Texas at Austin
}

This communication is the fifth in a series of largely descriptive papers dealing with the mating behavior of cockroaches (see Barth, 1961; 1964; 1968a \& b; Roth and Barth, 1967). The aim of this series is twofold: first to provide background information for experimental studies, and second to provide the detailed comparative information necessary for a study of the evolution of mating behavior within the Blattaria. A more general introduction to the series may be found in Barth (1964). The mating behavior of the Florida Woodroach, Eurycotis floridana (Walker) forms the subject of this communication.

\section{MATERIALS AND METHODS}

Stock cultures of $E$. floridana were maintained as described by Barth (1964) for Byrsotria fumigata. The observations on mating behavior were made in the evening (the normal active period for these animals) under red illumination in specially designed observation chambers (for details, see Barth, I964). The ethological terms employed in the description of the behavior patterns have been previously defined by Barth (1964).

\section{RESULTS AND DISCUSSION}

Eurycotis floridana is a rather large ( 3 to $4 \mathrm{~cm}$. in length), relatively robust cockroach of deep reddish brown coloration, found in Florida and other southeastern states, generally in woodlands under logs and debris. The sexes are similar in appearance except for the external genitalia. Both sexes are wingless with reduced tegmina extending no farther than the posterior border of the mesothoracic segment. E. floridana along with other members of this genus emits when alarmed an odoriferous secretion from a sternal scent gland

\footnotetext{
${ }^{1}$ No. 5 in a series of papers entitled "The Mating Behavior of Cockroaches".

${ }^{2}$ Much of this work was carried out at the Biological Laboratories, Harvard University. Financial support from National Science Foundation Predoctoral Fellowships and N. S. F. Grant G 19962 is gratefully acknowledged.
}

Manuscript received by the editor June 24, 1968 
located between the sixth and seventh abdominal sternites. The secretion which is produced only by adults, consists chiefly of trans 2-hexenal and has been shown to be an effective deterrent for certain potential predators (Roth, Niegisch, \& Stahl, 1956; Stay, I957; Dateo and Roth, 1967).

Roth and Willis (1954) have made a few observations on the mating behavior of E. floridana. According to their account, the male stands near the female repeatedly vibrating his body from side to side and extending his abdomen slightly, revealing the light colored intersegmental membrane between the sixth and seventh tergites. The female then applies her mouthparts to the male's dorsum, starting near the posterior tip of the abdomen and gradually working forward, straddling his abdomen, until the first abdominal tergite (on which is located a small glandular area bearing a patch of setae) is reached. After genital connection is achieved, the pair assume the opposed position characteristic of cockroaches in copula.

\section{Description of Normal Mating Behavior}

The following description of the mating behavior of $E$. floridana is based on observations of five successful copulations and numerous unsuccessful copulation attempts. The mating behavior of this species, particularly the early stages of the sequence, differs markedly from that considered typical for cockroaches. (For a description of the typical pattern, see Barth, I968c). One obvious difference is, of course, the absence of a wing raising display in the male. Owing to the extreme variation in the sequence of activities during mating behavior in this species, it has been impossible from the limited amount of data available to determine with any degree of certainty the releasers of the various activities in the sequence, or even to determine the usual sequence. For this reason, a slightly different procedure from that employed heretofore (Barth, 1964; 1968a \& b; Roth \& Barth, 1967) will be followed in the discussion of the mating behavior of this species. First a courtship sequence which led to a successful copulation will be described. Then some of the differences observed in other sequences will be discussed. Finally an attempt will be made to draw what conclusions concerning releasers can be drawn from the limited amount of data available.

I. A single mating behavior sequence

This particular sequence was initiated by the female approaching the male. Each animal waved its antennae back and forth vigorously and repeatedly stroked the other's body with them. The abdomen 
of the male was extended somewhat so that the intersegmental membranes between abdominal tergites one and two and seven and eight were exposed. The male then displayed the lateral jerking or vibration movement described by Roth and Willis (1954). This movement resembled the lateral jerking which often precedes aggressive encounters in Periplaneta (Barth, 1968a), except that each burst of movement usually consisted of five to ten small amplitude side to side swings of the body rather than two or three, as in Periplaneta. The movement seemed to result from alternate flexion and extension of the legs (particularly the femur-tibial joint) of the two sides during which the tarsi remained firmly planted on the substratum. These bursts of lateral vibration were generally performed two or three times in succession, with brief rest periods of from one to three or four seconds between them. Then after a long rest period of from 15 to 30 seconds, the series of bursts was repeated, each burst lasting about a second. During lateral vibration the abdomen was often extended somewhat more, so that the intersegmental membrane between tergites six and seven also became visible. It is worth noting that not all males expose the intersegmental membranes during vibration. Membrane exposure may be related to the level of sexual motivation in the particular individual concerned.

After several sets of vibration bursts, the female mounted the male actively and performed feeding movements for nearly a minute on the surface of the first two abdominal tergites. In this particular sequence, the female mounted from the side and, though the male made feeble attempts to twist around underneath the female, no genital connection was achieved. While the female was mounting, the male extended his abdomen considerably and pressed it against the substratum. The phallomeres were protruded partially from time to time. During mounting and copulation attempts, the antennae of both pair members oscillated vigorously. The female finally slipped off and the two faced each other with heads touching and antennae waving slowly for about two minutes. The male held his abdomen off the substratum and showed bursts of lateral vibration every few seconds. Four times during this period the female responded to the male's vibration with lateral vibration bursts of her own. The genital opening of the female gaped widely, which from a number of observations appears to be characteristic of receptive females. The male then ceased vibrating, moved back a few steps, and turned $90^{\circ}$, so that he was oriented laterally to the female. The female attempted to mount but was knocked off by a third individual passing by. The male vibrated again and turned another $90^{\circ}$, 
so that his abdominal tip was near the female's head. This turning or pivoting of the male between series of lateral vibration bursts appears to be quite characteristic of this species. At this point, the female mounted again and fed vigorously in the vicinity of the first two abdominal tergites, though occasionally her mouthparts wandered as far forward as the prothorax. The abdomen of the male was markedly extended and depressed against the substratum, and the phallomeres were protruded rhythmically. After another minute during which the female twice slipped off to the side and remounted, the union was accomplished, the female slipping off to one side and swinging around $180^{\circ}$. Internal twisting of the male's genitalia must have occurred during the turning movement, as there was no sign of abdominal twisting during or after the assumption of the opposed position. The female's genital opening gaped so widely during copulation that the whole tip of the male's abdomen appears to be inserted, as is shown in Plate 3 of Roth and Willis (1954).

\section{General observations on mating behavior in E. foridana}

Surprisingly enough, the female clearly seems to be the initiator of courtship behavior in this species. She was the initiator in virtually all the mating sequences observed. In the mating behavior observation chamber experiments, a number of females invariably rushed into the male side of the chamber as soon as the partition was removed. By contrast, the males usually remained motionless, though sometimes a few slowly wandered into the female side of the chamber. Even males showing lateral vibration and therefore presumably sexually motivated, made no effort to seek out females. Rather, they remained stationary, letting the females approach and mount before attempting copulation. Females tended to mount males quite indiscriminately whether the latter were showing the vibration movement or not. Generally, non-vibrating males remained entirely passive when mounted by a female, in which case the female usually departed after a minute or two of feeding, but occasionally they responded aggressively to mounting attempts by jerking the abdomen upward and/or kicking back with the metathoracic legs, or more rarely, they responded by running out from under a mounting female. In the latter case, the female often chased the escaping male for a short distance, attempting to remount. Generally, only those males which have shown the lateral vibration movement attempt to copulate with a mounting female. Only a single case was observed in which a male mounted by a female attempted copulation without having previously shown lateral vibration. Occasionally a female may 
MATING BEHAVIOR OF EURYCOTIS FLORIDANA

१ BEHAVIOR

RELEASER

$\sigma^{x}$ BEHAVIOR

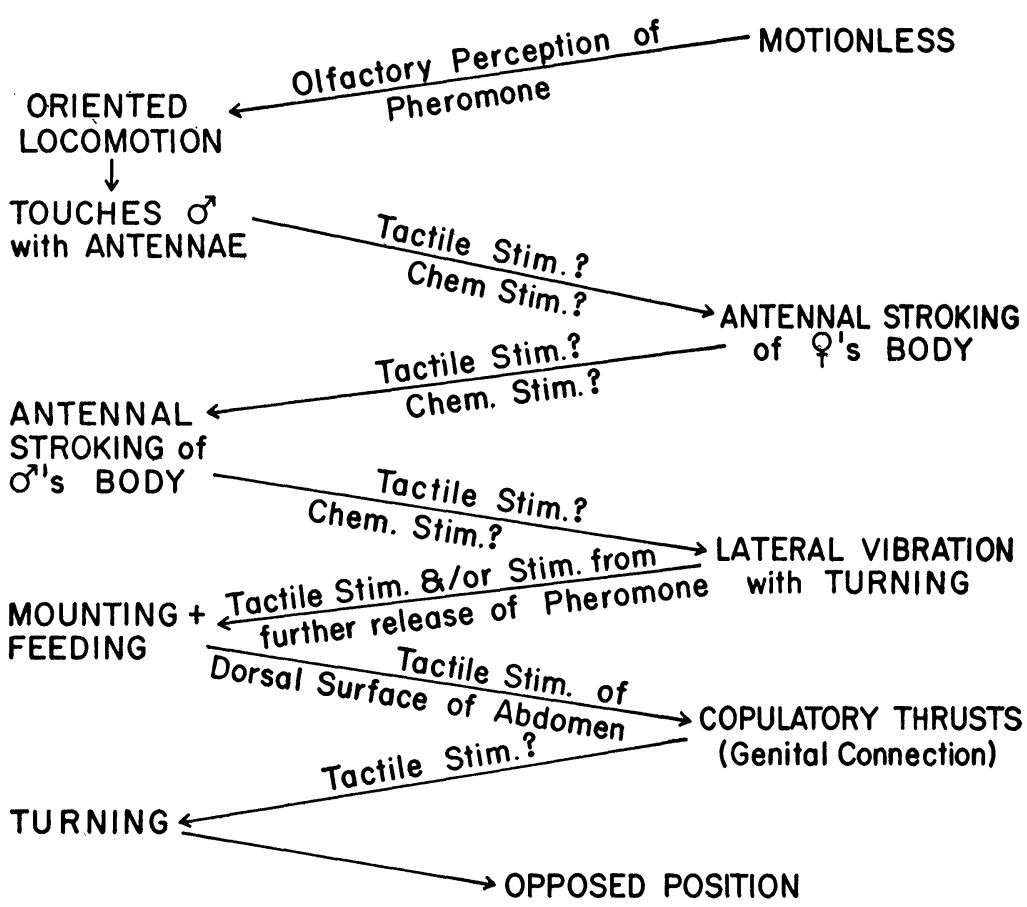

Figure 1. A summary of the mating behavior of Eurycotis floridana indicating the possible releasers for each step in the sequence.

mount another female, but this is much less likely to be tolerated by the female being mounted than by a male under similar circumstances. Females subjected to mounting by other females either run out from under or respond aggressively showing the behavior patterns of sexually unreceptive males. The orientation of mounting movements in this species is often very poor. The female may mount from any angle, but copulation is rarely successful unless she mounts from the correct posterior position, as males rarely attempt to perform the lateral movements necessary to correct a poorly oriented mounting.

Copulating pairs remain quiescent in the opposed position unless disturbed by the courting activities of other individuals. Such dis- 
turbances are frequent in the mating chambers and usually result from females attempting to mount and feed on the male's abdominal surface. In these cases both members of the pair show repeated vertical jerks of the abdomen, either alternately or in unison. If this does not discourage the courting individual, the pair resorts to lateral kicking. On several occasions, a copulating pair was observed to turn on their sides as though trying to dump the intruder off. This turning appears to be initiated by the female and results from the full extension of the legs on the opposite side. However, this movement may not necessarily result from the interference of other animals, as it has been observed on several occasions in copulating pairs which were not being disturbed by any other animal. If the pair shows any locomotion during copulation, the female predominates, dragging the male along behind in the manner characteristic of cockroaches.

\section{Homosexual and Pseudofemale Behavior}

Spontaneous male-male courtship sequences appear to be quite rare in this species, though they have been observed on two occasions. Male-male courtship sequences have also been observed upon exposure of isolated males to virgin females, though it is not certain that the frequency of such sequences is increased appreciably in this situation as it is in most cockroach species (Barth 1964; 1968a, Roth \& Barth 1967). Unlike females, males only mounted males which were performing the vibration movement. With this single exception, male-male courtship sequences do not appear to differ from male-female unsuccessful copulation attempts.

The Role of Various Releasers in the Courtship Sequence

A tentative schematic representation of the mating behavior of $E$. floridana indicating the possible releasers of the various stages in the sequence is shown in Fig. I. This diagram presents a relatively simple version of the mating behavior of Eurycotis. Some sequences do indeed follow this pattern, but departures from it are so frequent that one can't be sure that it represents an accurate picture of this: species' mating behavior. The suggested releasers are quite speculative, though there is some circumstantial evidence for some of them, as will be discussed below.

As we have seen, the female appears to be the usual initiator of courtship in this species. The most plausible explanation for the release of active courting behavior in females upon their exposure to males is that the males are producing a volatile pheromone which 
attracts females. Both contact chemoreception of this hypothetical substance and tactile stimulation resulting from contact with a male may be involved in the release of subsequent mounting behavior. This male sex pheromone must be produced by inactive males, as females approach and mount actively before males show any signs of sexual arousal.

The most puzzling feature of the courtship of this species is the lateral vibration movement, which characteristically appears in males after a brief exposure to courting females. The stimuli involved in the release of this movement, its function, and its possible motivational basis remain obscure. Observations indicate that this movement appears in a bewildering variety of situations within the general context of mating behavior. It occurs most frequently in isolated males upon exposure to virgin females which are actively engaged in mounting attempts. Under these circumstances, it may appear in males which are being mounted by females, in males which are being touched by the antennae of females, and occasionally in males which have no contact with another animal at all. In the latter case, the possible role of contact with a virgin female just previous to the observed display can not be discounted. Lateral vibration may be shown by a male which is mounting another vibrating male. It frequently appears in the male upon separation of a pair after an unsuccessful copulation attempt. It has also been observed in males which were attempting to escape from mounting females. Once or twice it has been observed in isolated males in the absence of females. This movement also occurs, though less commonly, in females, in which it is confined to two situations: a) in situations preceding mounting, in which the female has made antennal contact with the antennae of a vibrating male, and b) sometimes after unsuccessful copulation attempts.

The nature of the stimuli involved in the release of lateral vibration remains uncertain, but it seems unlikely that it is normally released by tactile stimulation alone, although the latter is evidently sufficient in a few instances as the display occasionally appears in isolated males. There is no satisfactory evidence that a volatile pheromone is involved, though the display is sometimes given by animals lacking contact with other animals. It was not possible to release this movement in isolated males by providing them with pieces of filter paper which had lined containers of virgin females for varying periods of time. Animals which accidentally wander onto such pieces of filter paper occasionally examine them with their antennae and palps (holding the maxillary palps against the 
surface of the paper in a stationary fashion rather than jittering them as in Periplaneta, Barth 1968a), but never show any additional signs of sexual motivation. Chemoreception of some substance present on the body of the virgin female, and also tactile stimulation from contact with the female are probably the usual releasers of lateral vibration. However as we have seen, it sometimes appears in situations lacking certain of these stimuli. Differences in the balance of tendencies making up the motivational state of the animal concerned may be involved in such cases.

The fact that lateral vibration appears in so many different situations in E. floridana makes it very difficult to make inferences concerning its motivational basis. It appears to be similar in form to the lateral jerking movement of Periplaneta (Barth, 1968a) and may well be homologous with it. In Periplaneta, lateral jerking appears in agonistic situations and it seems to indicate a fairly high level of escape tendency, in addition to a certain level of attack tendency. This is based on the observation that $P$. americana individuals showing the lateral jerking movement frequently flee if the animal toward whom the display is directed, rather than being intimidated, responds with a similar movement or with some higher intensity aggressive movement such as lateral kicking or a biting attempt. The appearance of this movement in E. floridana in some of the situations mentioned above is somewhat clarified by assuming a certain underlying level of both attack and escape tendencies in the animal performing it. However, in this species, the movement has taken on a sexual function, and presumably is indicative of a certain level of sexual motivation as well, as it almost always precedes copulatory movements in the male. This species may represent an intermediate stage in the evolution of the lateral vibration display, in which a sexual component has been added to its ancestral agonistic motivational basis. However, the possibility that subtle differences (perhaps in the nature of the tactile or olfactory stimuli presented) exist among vibration displays appearing in different contexts, i.e., that two or more distinct displays are involved, can not be overlooked.

In spite of the fact that females fairly frequently mount males which are not showing lateral vibration, the major function of this display appears to be the release of mounting and feeding behavior. Females in antennal or body contact with males showing lateral vibration almost invariably mount, and males apparently mount only vibrating males. Females have also been observed to mount vibrating females. Although tactile stimulation is probably of importance in 
the release of mounting behavior in animals in contact with a vibrating animal, it is evidently not the only source of stimulation involved, as females have been observed running from a distance of several inches and mounting a vibrating male directly without previous contact. Visual clues seem unlikely to be involved in the release of mounting and feeding by lateral vibration. It seems likely that some sort of pheromone stimulation is involved, perhaps the same as that which originally attracted the female. The displaying animal may release a greater quantity of the pheromone than a nondisplaying one or the movements may set up air currents which would serve to disseminate the pheromone, a function also suggested for the wing fluttering of displaying males of Periplaneta (Barth, I968a). The question of whether this hypothetical male sex pheromone is the same as the substance produced by the glandular area on the first abdominal tergite upon which the mounting female feeds awaits further work. The importance of this lateral vibration movement in the courtship of the male is emphasized by the fact that males rarely attempt copulation with mounting females unless the males have previously shown the vibration display. Thus lateral vibration may be considered to be an indication of sexual receptivity in the male.

The tactile stimulation of the dorsal abdominal surface of the male resulting from female's mounting and feeding activities is in all likelihood the major releaser of the male's copulatory movements. A considerable amount of stimulation seems to be required, as the mounting and feeding phase of the mating behavior sequence is more prolonged in E. floridana than in any other species thus far. Presumably tactile stimulation resulting from genital contact releases the turning movements which result in the assumption of the opposed position.

\section{CONCLUSION}

The mating behavior of this species is quite aberrant, differing considerably from that of other blattids which have been studied (all members of the Blattinae - Barth, I968a). Many of these differences are doubtless the result of morphological specializations (winglessness), and the active role of the female in courtship. These differences include the curious lateral vibration movement and the absence of wing raising and backing movements in the male, and the prolonged period of feeding in the female. Possible differences in releasers between E. floridana and other Blattidae have already been discussed. It is obvious that further study of this peculiar species is necessary before its mating behavior can be fully understood. Furthermore, 
study of the mating behavior of additional members of the Polyzosteriinae would be highly desirable, for such a study might reveal intermediate stages in the evolution of the aberrant mating behavior pattern found in E. floridana and thus aid in our understanding of this pattern.

\section{SUMMARY}

The mating behavior of Eurycotis floridana shows a considerable departure from the pattern typical of cockroaches. The female initiates the sequence in this species by approaching the male from a short distance. The male sex pheromone is somewhat volatile and serves the function of sex recognition and attraction of members of the opposite sex. Contact is established by the female and this is followed by antennal fencing and mutual body stroking with the antennae. Receptive males show a curious side to side rocking movement termed lateral vibration. The female mounts directly at this point without any further display on the part of the male. Since the male is wingless, the dorsum of his abdomen with a tergal gland on the first segment is freely accessible to the female. The mounting and feeding activities of the female are unusually prolonged in this species. Tactile stimulation of the abdominal dorsum releases the copulatory thrusts of the male. Genital connection is followed by the assumption of the opposed copulatory position.

BARTh, ROBERT H. JR.

\section{REFERENCES}

1961. Comparative and Experimental Studies on Mating Behavior in Cockroaches. Ph.D. Thesis, Harvard University, Cambridge, Massachusetts, 274 pages.

1964. The mating behavior of Byrsotria fumigata (Guérin) (Blattidae, Blaberinae). Behaviour 23: 1-30.

1968a. The mating behavior of Periplaneta americana (Linnaeus) and Blatta orientalis Linnaeus (Blattaria, Blattoidea, Blattidae, Blattinae) with notes on the mating behavior of three additional species of Periplaneta and on the interspecific effectiveness of the female sex pheromones of cockroaches. ms. submitted to Behaviour.

1968b. The mating behavior of Gromphadorhina portentosa (Schaum) (Blattaria, Blaberoidea, Blaberidae, Oxyhaloinae): an anomalous pattern for a cockroach. Psyche 75: 124-131.

1968c. The comparative physiology of reproductive processes in cockroaches. Part I. Mating behavior and its endocrine control. Advances in Reproductive Physiology 3: 167-207.

Dateo, George P. \& L. M. Roth

1967. Occurrence of gluconic acid and 2-hexenal in the defensive secretions of three species of Eurycotis (Blattaria: Blattidae: Polyzosteriinae). Ann. Ent. Soc. Am. 60: 1025-1030. 
Roth, Louis M. AND R. H. Barth JR.

1967. The sense organs employed by cockroaches in mating behavior. Behavior 28: 58-94.

Roth, Louis M., W. D. Niegisch, and W. H. Stahl

1956. Occurrence of 2-hexenal in the cockroach, Eurycotis floridana Science 123: 670-671.

Roth, Louis M. AND E. R. Willis

1954. The reproduction of cockroaches. Smith, Misc. Coll. 122(12): 1-49.

StAY, Barbara

1957. The sternal scent gland of Eurycotis floridana (Blattaria: Blattidae) Ann. Ent. Soc. Am. 50: 514-519. 

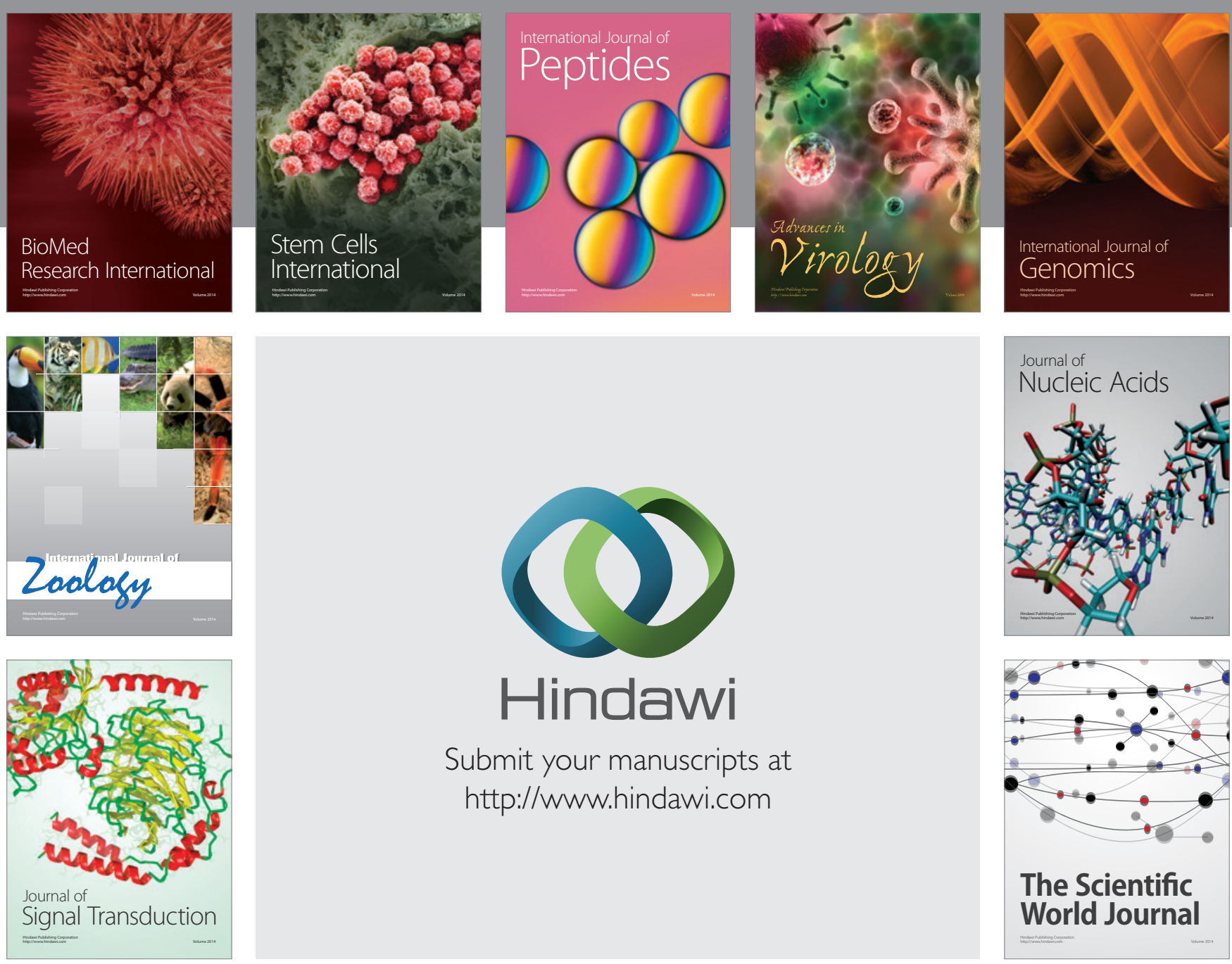

Submit your manuscripts at

http://www.hindawi.com
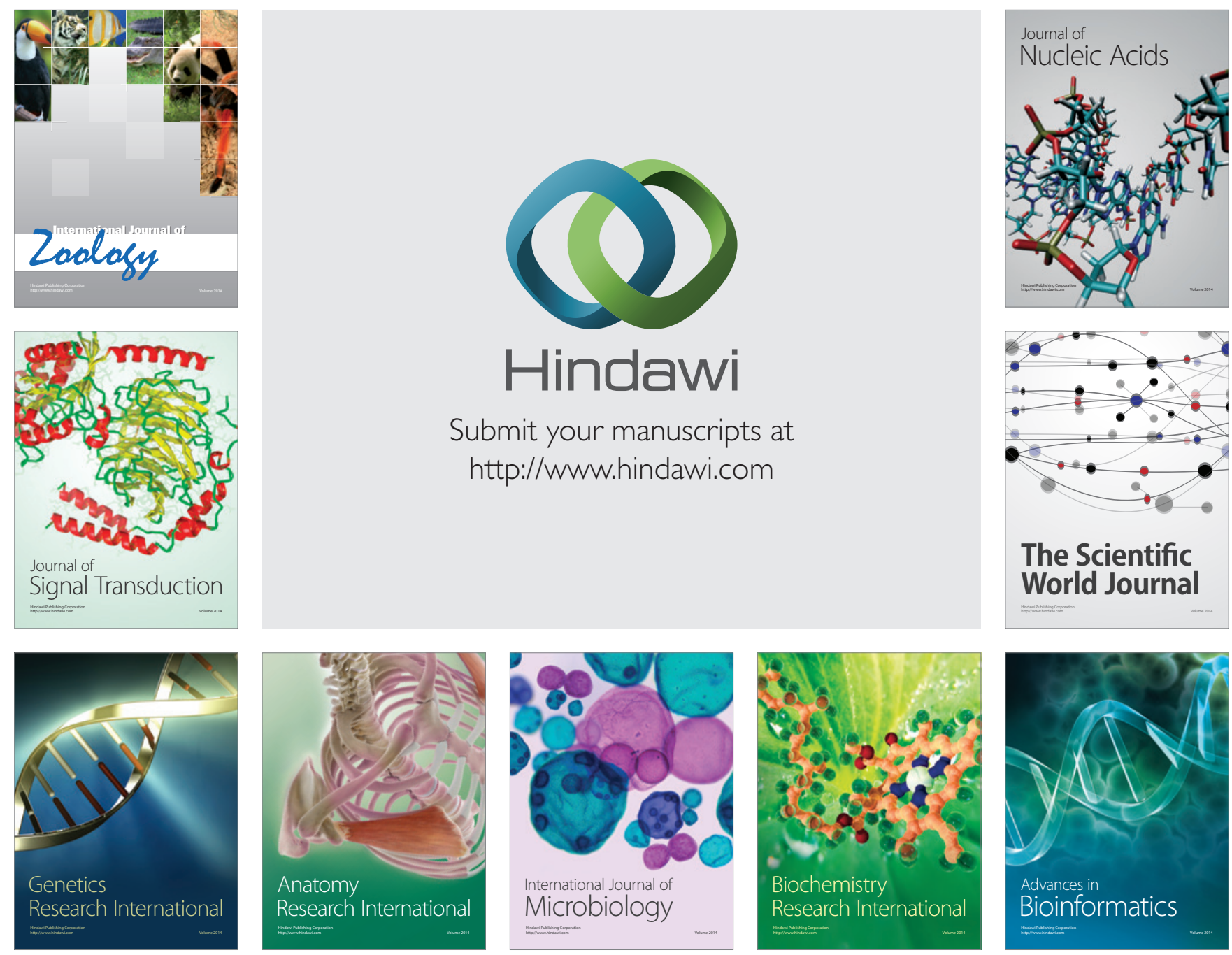

The Scientific World Journal
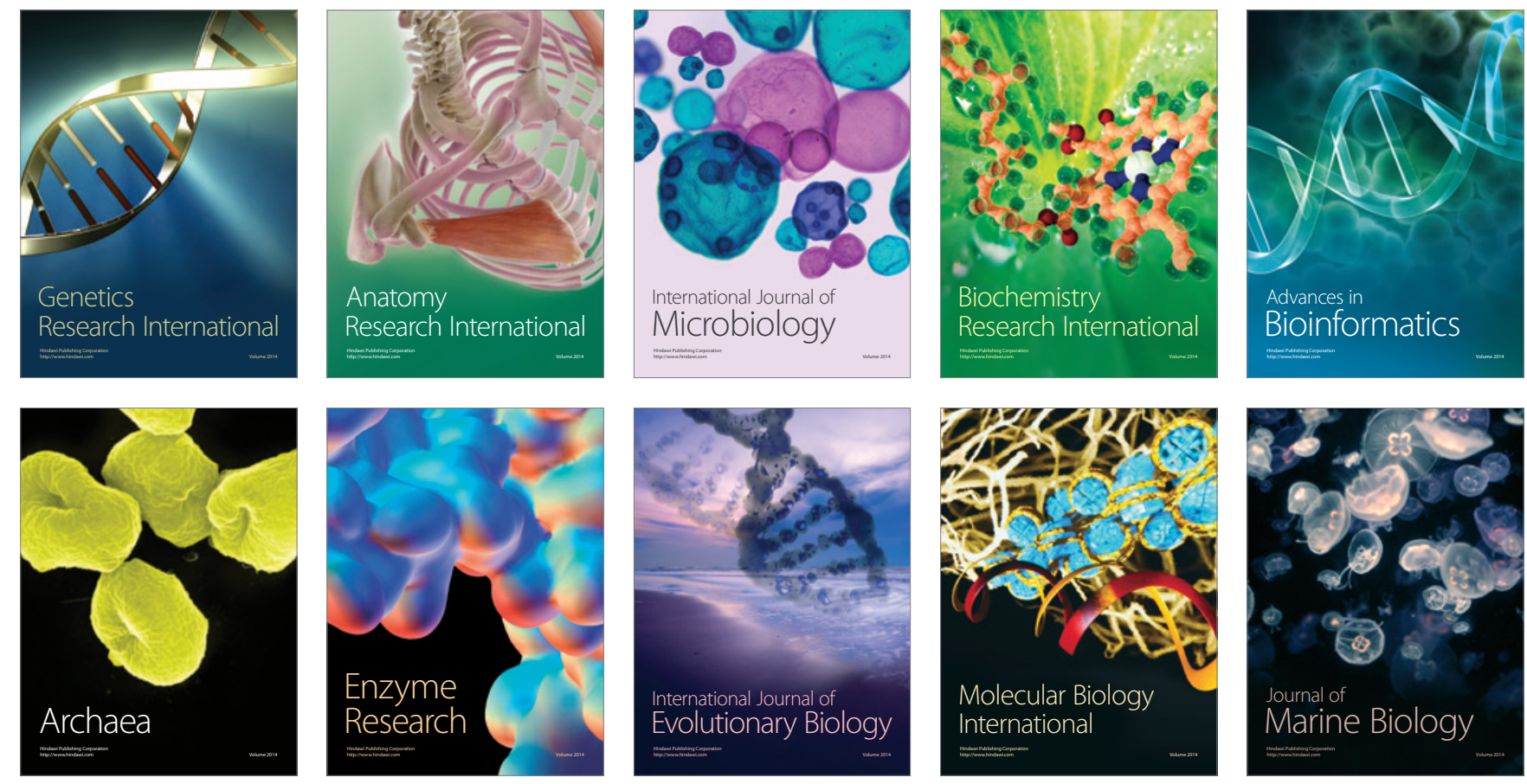